\title{
The benefits of inositol-stabilized arginine silicate as a workout ingredient
}

\author{
S Rood-Ojalvo ${ }^{*}$, D Sandler², E Veledar ${ }^{3}$, J Komorowski ${ }^{1}$ \\ From The Twelfth International Society of Sports Nutrition (ISSN) Conference and Expo \\ Austin, TX, USA. 11-13 June 2015
}

\section{Background}

The purpose of this study was to examine the benefits of inositol-stabilized arginine silicate (ASI; Nitrosigine ${ }^{\circledR}$ ) as a workout ingredient in healthy adults. ASI has been previously shown to significantly enhance blood levels of arginine up to six hours post-dose and increase nitric oxide levels. To investigate reports of enhanced energy, increased muscle pump and stamina during workouts, and faster muscle recovery post-workout, ASI $(1,500 \mathrm{mg} /$ day) was tested in a double-blind placebo-controlled crossover-design (DBPC-X) study using the POMS vigor-activity and fatigue-inertia sub-scores, blood flow measurements, leg circumference measurements, and biomarkers of muscle recovery (creatine kinase (CK) and lactate dehydrogenase (LDH)) as outcome measures.

\section{Methods}

The DBPC-X study was conducted in male subjects $(\mathrm{N}=$ 16 per group) who had limited exercise routines prior to participating in the study. These subjects took ASI daily for 4 days. Subjects had baseline measurements drawn at the hour 0 visit, took the study product, and completed an intense leg extension exercise protocol to induce muscle soreness. Subjects returned after 24, 48 , and 72 hours for additional study measurements. After 72 hours, subjects repeated the leg extension exercise protocol. There was a seven-day washout between test products. Between-product assessments were primary endpoints and within-product assessments secondary endpoints.

\section{Results}

Sixteen (16) healthy male subjects (19-33 years of age) completed the study. Perceived energy, measured using the POMS vigor-activity sub-scores, significantly increased

\footnotetext{
* Correspondence: sroodojalvo@nutrition21.com

1 Nutrition 21, LLC, Purchase, Harrison, NY, USA

Full list of author information is available at the end of the article
}

after 72 hours compared to placebo $(\mathrm{p}=0.039)$. At 72 hours, perceived fatigue, measured using the POMS fatigue-inertia sub-scores, significantly decreased in the ASI group ( $\mathrm{p}=0.041$ ) from pre-dose, compared to a non-significant change in the placebo group ( $\mathrm{p}=0.580) ; \mathrm{p}=0.055$ between groups.

Hyperemia, measured using leg circumference, increased significantly in the ASI group by $1.8 \mathrm{~cm}(\mathrm{p}=0.001)$ at 72 hours from pre-dose, compared to a non-significant increase in the placebo group by $0.8 \mathrm{~cm}(\mathrm{p}=0.091)$; $\mathrm{p}=0.070$ between groups.

Blood flow, measured by blood velocity through the femoral artery using a Doppler Ultrasound, increased $59.9 \mathrm{~cm} / \mathrm{s}$ in the ASI group $(\mathrm{p}<0.005)$ and $49.9 \mathrm{~cm} / \mathrm{s}$ in the placebo group $(\mathrm{p}<0.005)$ after exercise on Day 3; $\mathrm{p}=0.2$ between groups.

CK levels significantly decreased in the ASI group at 24 $(\mathrm{p}=0.040), 48(\mathrm{p}=0.017)$ and $72(\mathrm{p}=0.034)$ hours postexercise compared to the placebo group. Immediately post-exercise at the hour 0 visit, ASI supplementation led to $44 \%$ less muscle damage, measured by CK levels, than placebo $(p=0.057)$. LDH levels significantly increased from baseline immediately after exercise in the placebo group ( $\mathrm{p}=0.015)$, but not in the ASI group $(\mathrm{p}=0.366)$; $\mathrm{p}=0.133$ between groups. No safety concerns were raised by this study.

\section{Conclusion}

Both primary and secondary endpoints show that daily doses of ASI prior to workout significantly increased preworkout energy levels, increased muscle pump immediately following a workout, and decreased biomarkers of muscle damage immediately after a workout and during recovery. These results demonstrate multiple benefits of ASI as a functional workout ingredient. 


\title{
Authors' details
}

'Nutrition 21, LLC, Purchase, Harrison, NY, USA. ${ }^{2}$ StrengthPro Inc, Golden, CO,

USA. ${ }^{3}$ Emory University, Atlanta, GA, USA.

Published: 21 September 2015

\author{
doi:10.1186/1550-2783-12-S1-P14 \\ Cite this article as: Rood-Ojalvo et al:: The benefits of inositol-stabilized \\ arginine silicate as a workout ingredient. Journal of the International \\ Society of Sports Nutrition 2015 12(Suppl 1):P14.
}

Submit your next manuscript to BioMed Central and take full advantage of:

- Convenient online submission

- Thorough peer review

- No space constraints or color figure charges

- Immediate publication on acceptance

- Inclusion in PubMed, CAS, Scopus and Google Scholar

- Research which is freely available for redistribution

Submit your manuscript at www.biomedcentral.com/submit 